Bulletin of the Section of Logic

Volume 47/3 (2018), pp. 201-214

http://dx.doi.org/10.18778/0138-0680.47.3.05

Mateusz Klonowski

\title{
A POST-STYLE PROOF OF COMPLETENESS THEOREM FOR SYMMETRIC RELATEDNESS LOGIC S*
}

\begin{abstract}
One of the logic defined by Richard Epstein in a context of an analysis of subject matter relationship is Symmetric Relatedness Logic S. In the monograph [2] we can find some open problems concerning relatedness logic, a Post-style completeness theorem for logic $\mathrm{S}$ is one of them. Our paper introduces a solution of this metalogical issue.
\end{abstract}

Keywords: normal forms, Post-style proof of completeness, relatedness logic, relating logic.

\section{The Epstein's logics}

In the case of most of non-classical interpretations of conditionals two aspects are considered as substantial:

1. logical values of an antecedent and a consequent

2. a relationship between an antecedent and a consequent.

The analysis of conditionals introduced by Richard Epstein in [2] are based on ways of understanding of relationships postulated by 2 . Different concepts allow for the presentation of different implications. In order to define truth conditions for these logical connectives some binary relations based on a set of formulas with some constraints are introduced. But Epstein introduces also a different approach. He considers some functions which

* The author would like to thank the National Science Centre of Poland for the support of this research through grant No.: UMO-2015/19/N/HS1/02401 and Tomasz Jarmużek for his remarks and suggestions. 
assigns to each formula some set (set-assignments). Such functions are intended to enable a notion of a content (subject matter) on a formal ground to be represented. In this case it is important to consider relations between contents of propositions, for instance one might be contained in another. These two approaches are proved to be equivalent in the case of some logics introduced by Epstein. He considered two families of logics defined by classes of models with only one relation. Namely relatedness logics and dependence logics [2, pp. 61-84, 115-143].

Epstein defined two relatedness logic. Symmetric Relatedness Logic S is one of them. Among problems analysed in [2] there is a question about a Post-style proof of completeness theorem for logic S. In this paper a solution of this metalogical issue will be presented.

The Epstein's approach via set-assigments appeared to be quite fruitful for expressing many intensional logics like modal logics, intuitionistic logic, many-valued logics or paraconsistent logics [2, pp. 145-287]. That is why Stanisław Krajewski proposed to treat the analysis of Epstein as a bigger project concerning logics of two aspects of propositions: their logical value and contents (see [4, pp. 17-18]). The general concepts and the most important results of such approach has been presented in [1], [2], [4] and also [5].

A much different line is examined in [3] by Jarmużek and Kaczkowski. In this case authors consider a logic defined by models with one binary relation without any extra constraints. However, in this approach only two intensional connectives: implication and conjunction were examined.

Now, more extensive research on this kind of logic, but with the language consists of all Boolean connectives and intensional counterpart of binary Boolean connectives, is being done, since any binary connective can be interpreted by logical valuation of components and binary relation. Presently, we can distinguish various relational conditions that may determine subclasses of the class of all binary relations of formulas, and consequently define numerous logics of the considered kind. Any of such logic is called relating logic. ${ }^{1}$ In consequence, Epstein approach is a special case of relating logics program, since Epstein's logics are special cases of relating logics. Note that the converse dependence does not hold. It is

\footnotetext{
${ }^{1}$ The ideas concerning relating logic were developed during a logic seminar held in Torun, led by Tomasz Jarmużek and they are in various forms being studied, examined and developed by Torunian $\mathrm{PhD}$ students, participating in that seminar.
} 
also worth noticing that an analysis of relating logics seems to be promising for a philosophical interpretation of relating connectives like causal or temporal ones. Such issue should be a subject of the further investigations concerning applications of relating logics.

\section{Language of relatedness logic}

Formulas of relatedness logic are build by means of propositional letters $p_{1}, p_{2}, \ldots$, three logical connectives $\neg, \wedge, \rightarrow$ and parentheses $),($. A set of propositional letters is denoted by $\mathrm{Pl}$. A set For of formulas is the smallest set $\Sigma \subseteq$ PI such that: if $A \in \Sigma$, then $\neg A \in \Sigma$ and if $A, B \in \Sigma$, then $(A \wedge B),(A \rightarrow B) \in \Sigma$. We will omit the outermost parentheses. In the case of formulas build by an iteration of $\wedge$ we shall agree to associate to the left and write, for instance, $A \wedge B \wedge C$ instead of $(A \wedge B) \wedge C$. In some cases we use parentheses ], [ in order to make some formulas and metalogical expressions more readable. Additionally to simplify some of formalism we introduce the following abbreviations for every $A, B, A_{1}, \ldots, A_{n} \in$ For $(n \geq 2)$ :

$$
\begin{aligned}
& A \leftrightarrow B:=(A \rightarrow B) \wedge(B \rightarrow A) \\
& A_{1} \vee \ldots \vee A_{n}:=\neg\left(\neg A_{1} \wedge \ldots \wedge \neg A_{n}\right) \\
& A \hookrightarrow B:=A \rightarrow(B \rightarrow B) \\
& A \supset B:=\neg(A \wedge \neg B) \\
& A \equiv B:=\neg(A \wedge \neg B) \wedge \neg(B \wedge \neg A) .
\end{aligned}
$$

By the complexity of a given formula we understand an output of function $c$ : For $\longrightarrow \mathbb{N}$ defined in a standard way, wherein $c(A)=0$, if $A \in \mathrm{Pl}$. A notion of subformula of a given formula is determined by function sub: For $\longrightarrow \mathcal{P}$ (For) also defined in a standard way. In order to refer to propositional letters of a given formula we use the following set $\mathrm{pl}(A)=\operatorname{sub}(A) \cap \mathrm{Pl}$, for every $A \in$ For.

\section{Notion of relatedness}

According to Epstein's analysis of relatedness there are at least two good candidates for formal attributes of a content relationship. The first one is reflexivity, motivated by the obvious fact that any content is identical with 
itself. The second one is to be independent from logical connectives, which is motivated by the fact that connectives are syncategorematic. Another intuitive attribute might be symmetry. In this way, we come to the concept of symmetric relatedness relation:

Definition 3.1. Relation $R \subseteq$ For $\times$ For is symmetric relatedness relation (for short: srr) iff $R$ fulfils the following conditions for every $A, B, C \in$ For:

$$
\begin{aligned}
& R(A, A) \\
& R(A, \neg B) \text { iff } R(A, B) \\
& R(A, B \wedge C) \text { iff } R(A, B \rightarrow C) \\
& R(A, B \wedge C) \text { iff }[R(A, B) \text { or } R(A, C)] \\
& R(A, B) \text { iff } R(B, A) .
\end{aligned}
$$

In the monograph [2, pp. 65-68] it is presented how by means of srr one can express contents relationships recognised as relationships between propositions due to a common subject matter. For a simple illustration of a such relationship let us consider the following propositions:

1. If John is interested in logic, then John knows Post's proof of completeness for Classical Propositional Logic

2. John considers a notion of normal forms for formulas of Classical Propositional Logic.

There are many subject matters which are shared by 1 and 2 , one of them might be expressed as metalogical properties of Classical Propositional Logic.

The next fact determines a way of extending reflexive and symmetric relations defined on PI to srr (see [2, pp. 67-68]).

FACT 3.2. Let $\mathrm{Q} \subseteq \mathrm{PI} \times \mathrm{PI}$ be reflexive and symmetric relation. Let $R \subseteq$ For $\times$ For be an extension of $Q$ on For defined for every $A, B \in$ For in the following way:

$$
R(A, B) \text { iff } \exists_{x \in \mathrm{pl}(A)} \exists_{y \in \mathrm{pl}(B)} \mathrm{Q}(x, y) .
$$

Then $R$ is srr.

Proof: Assume all hypothesis. Let $A, B, C \in$ For.

- Ad. (re). Let $a \in \mathrm{pl}(A)$. By reflexivity of $\mathrm{Q}, \mathrm{Q}(a, a)$. Therefore, by $(\star), \mathrm{R}(A, A)$. 
- Ad. (srr1). We have: $\mathrm{R}(A, \neg B)$, by $(\star)$, iff $\exists_{x \in \mathrm{pl}(A)} \exists_{y \in \mathrm{pl}(\neg B)} \mathrm{Q}(x, y)$, by equality $\mathrm{pl}(\neg A)=\mathrm{pl}(A)$ and classical logic, iff $\exists_{x \in \mathrm{pl}(A)} \exists_{y \in \mathrm{pl}(B)} \mathrm{Q}(x, y)$, by $(\star)$, iff $\mathrm{R}(A, B)$.

- Ad. (srr2). We have: $\mathrm{R}(A, B \wedge C)$, by $(\star)$, iff $\exists_{x \in \mathrm{pl}(A)} \exists_{y \in \mathrm{pl}(B \wedge C)}$ $\mathrm{Q}(x, y)$, by equality $\mathrm{pl}(B \wedge C)=\mathrm{pl}(B \rightarrow C)$ and classical logic, iff $\exists_{x \in \mathrm{pl}(A)} \exists_{y \in \mathrm{pl}(B \rightarrow C)} \mathrm{Q}(x, y)$, by $(\star)$, iff $\mathrm{R}(A, B \rightarrow C)$.

- Ad. (srr3). We have: $\mathrm{R}(A, B \wedge C)$, by $(\star)$, iff $\exists_{x \in \mathrm{pl}(A)} \exists_{y \in \mathrm{pl}(B \wedge C)}$ $\mathrm{Q}(x, y)$, by equality $\mathrm{pl}(B \wedge C)=\mathrm{pl}(B) \cup \mathrm{pl}(C)$, iff $\exists_{x \in \mathrm{pl}(A)} \exists_{y \in \mathrm{pl}(B) \cup \mathrm{pl}(C)} \mathrm{Q}(x, y)$, by definition of union and classical logic, iff $\left[\exists_{x \in \mathrm{pl}(A)} \exists_{y \in \mathrm{pl}(B)} \mathrm{Q}(x, y)\right.$ or $\left.\exists_{x \in \mathrm{pl}(A)} \exists_{y \in \mathrm{pl}(C)} \mathrm{Q}(x, y)\right]$, by $(\star)$, iff $[\mathrm{R}(A, B)$ or $\mathrm{R}(A, C)]$.

- Ad. (sym). We have: $\mathrm{R}(A, B)$, by $(\star)$, iff $\exists_{x \in \mathrm{pl}(A)} \exists_{y \in \mathrm{pl}(B)} \mathrm{Q}(x, y)$, by symmetry of $Q$ and classical logic, iff $\exists_{y \in \mathrm{pl}(B)} \exists_{x \in \mathrm{pl}(A)} \mathrm{Q}(y, x)$, by $(\star)$, iff $\mathrm{R}(B, A)$.

It is easy to see that an extension received by condition $(\star)$ is unique.

\section{Symmetric Relatedness Logic S}

Definition 4.1. A model of relatedness logic based on srr (or simply a model) is the following ordered pair $\langle v, R\rangle$ such that:

- $v \in\{1,0\}^{\mathrm{PI}}$ is a valuation of propositional letters

- $R \subseteq$ For $\times$ For is srr.

A class of models is denoted by $\mathcal{M}$. Relation $\mathrm{R}$ (resp. valuation $v$ ) of model $\mathfrak{M} \in \mathcal{M}$ is denoted by $\mathrm{R}_{\mathfrak{M}}$ (resp. $v_{\mathfrak{M}}$ ). Now we define a notion of a truth in a model:

Definition 4.2. Let $\mathfrak{M} \in \mathcal{M}$ and $A \in$ For. $A$ is a truth in $\mathfrak{M}$ (for short: $\mathfrak{M}=A$ ) iff for every $B, C \in$ For:

$$
\begin{array}{ll}
v_{\mathfrak{M}}(A)=1, & \text { if } A \in \mathrm{PI} \\
\mathfrak{M} \not \models B, & \text { if } A:=\neg B \\
\mathfrak{M} \models B \text { \& } \mathfrak{M}=C, & \text { if } A:=B \wedge C \\
{[\mathfrak{M} \not=B \text { or } \mathfrak{M} \models C] \mathscr{G} R_{\mathfrak{M}}(B, C),} & \text { if } A:=B \rightarrow C .
\end{array}
$$

For every $\Sigma \subseteq$ For and $\mathfrak{M} \in \mathcal{M}$ in the case $\forall_{A \in \Sigma} \mathfrak{M} \models A$ we will write $\mathfrak{M}=\Sigma$. 
It is easy to observe that by Definition 4.2 the following abbreviation $A \vee B, A \supset B, A \equiv B$ denote respectively extensionally interpreted disjunction, conditional and biconditional.

Let us notice that formula $A \rightarrow B$ plays a special role in Epstein's investigations. It enables to express srr on the ground of the language of relatedness logic (see [2, pp. 77-78]):

FACT 4.3. Let $\mathfrak{M} \in \mathcal{M}$ and $A, B \in$ For. Then: $\mathfrak{M} \models A \leftrightarrow B$ iff $R_{\mathfrak{M}}(A, B)$. Proof: Assume all hypothesis

„” Let $\mathfrak{M}=A \leftrightarrow B$, so $\mathfrak{M} \models A \rightarrow(B \rightarrow B)$. Hence, $\mathrm{R}_{\mathfrak{M}}(A, B \rightarrow$ $B)$. Thus, by (srr2), (srr3) we get $\mathrm{R}_{\mathfrak{M}}(A, B)$.

„£" Let $\mathrm{R}_{\mathfrak{M}}(A, B)$. Hence, by (srr2), (srr3), $\mathrm{R}_{\mathfrak{M}}(A, B \rightarrow B)$. By (re) and because either $\mathfrak{M}=B$ or $\mathfrak{M} \not \models B$, we get $\mathfrak{M} \models B \rightarrow B$. Hence, either $\mathfrak{M} \not \models A$ or $\mathfrak{M} \models B \rightarrow B$. Therefore, $\mathfrak{M} \models A \rightarrow(B \rightarrow B)$. Thus, $\mathfrak{M}=A \rightarrow B$.

Definition 4.4. Let $\Sigma \cup\{A\} \subseteq$ For. Then:

- A is a semantic consequence of $\Sigma$ in $S$ (nota.: $\Sigma \models_{S} A$ ) iff $\forall_{\mathfrak{M} \in \mathcal{M}}(\mathfrak{M}$ $\models \Sigma \Longrightarrow \mathfrak{M} \models A$ ).

- $A$ is a tautology in $S$ (nota.: $\models_{S} A$ ) iff $\varnothing \models_{\mathcal{M}} A$.

In the next section we remind Hilbert-style formulation of $\mathrm{S}$.

\section{Axiomatization of logic S}

Axiom schemata of logic $\mathrm{S}$ are the following formulas, for every $A, B, C \in$ For (see [2, p. 80]):

$$
\begin{aligned}
& A \leftrightarrow A \\
& (B \leftrightarrow A) \rightarrow(A \leftrightarrow B) \\
& (A \rightarrow \neg B) \leftrightarrow(A \leftrightarrow B) \\
& (A \leftrightarrow(B \rightarrow C)) \leftrightarrow((A \rightarrow B) \vee(A \rightarrow C)) \\
& (A \rightarrow(B \wedge C)) \leftrightarrow(A \leftrightarrow(B \rightarrow C)) \\
& (A \wedge B) \rightarrow A \\
& A \rightarrow(B \rightarrow(A \wedge B)) \\
& (A \wedge B) \rightarrow(B \wedge A) \\
& A \leftrightarrow \neg \neg A \\
& (A \rightarrow B) \leftrightarrow(\neg(A \wedge \neg B) \wedge(A \rightarrow B))
\end{aligned}
$$


A Post-Style Proof of Completeness Theorem for Symmetric Relatedness. . 207

$$
\begin{aligned}
& A \rightarrow(\neg(A \wedge B) \rightarrow \neg B) \\
& \neg(A \wedge B) \rightarrow(\neg(C \wedge \neg B) \rightarrow \neg(A \wedge C)) \\
& \neg((A \rightarrow B) \wedge(A \wedge \neg B)) .
\end{aligned}
$$

Schemata $\left(a x_{1}\right)-\left(a x_{5}\right)$ are intended to give a syntactic characterization of srr. The rest of schemata characterize logical connectives in logic S. The only rule of inference is modus ponens:

$$
\frac{A, A \rightarrow B}{B} .
$$

We have the standard definition of the relation of syntactic consequence for S:

Definition 5.1. Let $\Sigma \cup\{A\} \subseteq$ For. Then:

- $A$ is a syntactic consequence of $\Sigma$ in $S$ (nota.: $\Sigma \vdash_{S} A$ ) iff there is a finite sequence of formulas $B_{1}, \ldots, B_{n}$ such that $B_{n}=A$ and for every $i \leq n$ at least one of the following conditions holds: (1) $B_{i}:=$ $\left(\mathrm{ax}_{1}\right), \ldots,\left(\mathrm{ax}_{13}\right)$, (2) $B_{i} \in \Sigma$ or (3) $\exists_{j, k<i} B_{k}:=B_{j} \rightarrow B_{i}$.

- $A$ is a thesis in $S$ (nota.: $\vdash_{S} A$ ) iff $\varnothing \vdash_{S} A$.

One of the metalogical problems of logic $\mathrm{S}$ raised by Epstein concerns a proof of completeness by means of Post's method [2, s. 81]. He noticed, however, that a non-constructive proof of completeness might be received by a simple modifcation of a proof presented for Dependence Logic D [2, pp. 81, 126-129].

Let us notice that for every axiom schemata $A, \models_{\mathrm{S}} A$ and for every $A, B \in$ For, $A, A \rightarrow B \models_{\mathrm{S}} B$. Hence we have, the following fact:

FACT 5.2 (Theorem of weak soundenss for $\mathrm{S}$ ). Let $A \in$ For. Then: $\vdash_{S}$ $A \Longrightarrow \models_{S} A$.

Let $\vdash_{\mathrm{CPL}}$ be the relation of syntactic consequence for $\{\neg, \wedge\}$-fragment of Classical Propositional Logic. According to an observation of Epstein (see [2, pp. 74-75]) we should be able to prove the following fact:

FACT 5.3. Let $A \in$ For. Then: $\vdash_{C P L} A \Longrightarrow \vdash_{S} A$.

Let us notice that the following formulas are these in logic S:

$$
\begin{aligned}
& A \supset(A \wedge A) \\
& (A \wedge B) \supset A \\
& (A \supset B) \supset(\neg(B \wedge C) \supset \neg(C \wedge A)) .
\end{aligned}
$$


Moreover the following rule of modus ponens for $\supset$ is derivable:

$$
\frac{A, A \supset B}{B} .
$$

Formulas (1)-(3) with rule (4) (for formulas $A, B, C$ build only by means of $\neg, \wedge$ ) enable to determine relation $\vdash_{\mathrm{CPL}}$ (see $[6$, pp. 12-46, 54-76]).

\section{Normal forms of formulas}

The set of literals is defined in a standard way $\mathrm{Li}:=\mathrm{PI} \cup \mathrm{nPI}$, where $\mathrm{nPI}:=\{\neg A \in$ For $\mid A \in \mathrm{PI}\}$. Additionally we define a set of related propositional letters $\mathrm{rPI}=\{A \rightarrow B \in$ For $\mid A, B \in \mathrm{PI}\}$ and a set of non-related propositional letters $\mathrm{nrPI}=\{\neg(A \leftrightarrow B) \in$ For $\mid A, B \in \mathrm{PI}\}$.

Definition 6.1. $A \in$ For is elementary disjunction (for short: ed) in the following cases:

(1) $A \in \mathrm{Li} \cup \mathrm{rPI} \cup \mathrm{nrPI}$

(2) $A:=B \vee C$, where $B$ is ed, and $C \in \mathrm{Li} \cup \mathrm{rPI} \cup \mathrm{nrPI}$.

Remark 6.2. Let us notice that by Definition $6.1 A$ is ed iff $A:=B_{1} \vee$ $\ldots \vee B_{n}(n \in \mathbb{N})$, where for any $i \leq n, B_{i} \in \mathrm{Li} \cup \mathrm{rPI} \cup \mathrm{nrPI}$. The equivalence might be also used in order to define ed.

A conjunctive normal form is defined in a standard way:

Definition 6.3. A $\in$ For is in conjunctive normal form (for short: cnf) in the following cases:

(1) $A$ is ed

(2) $A:=B \wedge C$, where $B$ is in cnf and $C$ is ed.

REMARK 6.4. Similarly to Remark 6.2, let us notice that by Definition 6.3 $A$ is in cnf iff $A:=B_{1} \wedge \ldots \wedge B_{n}(n \in \mathbb{N})$, where for any $i \leq n, B_{i}$ is ed. The equivalence might be also used in order to define cnf.

Let us define a function that enables to refer to an «opposite formula» of any:

Definition 6.5. Let' $: \mathrm{Li} \cup \mathrm{rPI} \cup \mathrm{nrPI} \longrightarrow \mathrm{Li} \cup \mathrm{rPI} \cup \mathrm{nrPI}$ be a function such that, for every $A \in \mathrm{Li} \cup \mathrm{rPI} \cup \mathrm{nrPI}$ we put:

$$
A^{\prime}= \begin{cases}\neg A, & \text { if } A \in \mathrm{PI} \cup \mathrm{rPI} \\ B, & \text { if } A \in \mathrm{nPI} \cup \mathrm{nrPI} \& A:=\neg B .\end{cases}
$$

Let us notice that: 
A Post-Style Proof of Completeness Theorem for Symmetric Relatedness. . 209

- $A^{\prime}$ is ed

- $\vdash_{\mathrm{S}} \neg A \equiv A^{\prime}$

- $\vdash_{\mathrm{S}}(\neg A \rightarrow C) \equiv\left(A^{\prime} \rightarrow C\right)$, for every $C \in$ For

- $A^{\prime \prime}=A$.

We also define a function that enables to refer to an «antecedent» or «consequent» of the given formula:

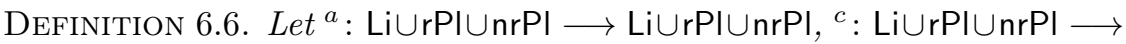
$\mathrm{Li} \cup \mathrm{rPI} \cup \mathrm{nrPI}$ be functions such that, for every $A \in \mathrm{Li} \cup \mathrm{rPI} \cup \mathrm{nrPI}$ we put:

$$
\begin{aligned}
A^{a} & = \begin{cases}A, & \text { if } A \in \mathrm{PI} \\
A^{\prime}, & \text { if } A \in \mathrm{nPI} \\
B, & \text { if } A \in \mathrm{rPI} \cup \mathrm{nrPl} \mathscr{E}[A:=B \rightarrow C \text { or } A:=\neg(B \rightarrow C)] .\end{cases} \\
A^{c} & = \begin{cases}A, & \text { if } A \in \mathrm{PI} \\
A^{\prime}, & \text { if } A \in \mathrm{nPI} \\
B, & \text { if } A \in \mathrm{rPI} \cup \mathrm{nrPl} \mathscr{E}[A:=C \rightarrow B \text { or } A:=\neg(C \rightarrow B)] .\end{cases}
\end{aligned}
$$

Let us notice that:

- $A^{a}, A^{c}$ are ed

- $\vdash_{\mathrm{S}}(A \rightarrow C) \equiv\left(\left(A^{a} \rightarrow C\right) \vee\left(A^{b} \rightarrow C\right)\right)$, for every $C \in$ For.

FACT 6.7. Let $A \in$ For. Then, there is $B \in$ For in cnf such that: $\vdash_{S} A \equiv B$ and for every $C \in$ For, $\vdash_{S}(A \leftrightarrow C) \equiv(B \rightarrow C)$.

Proof: We use induction on complexity of formulas.

Basis. Let $A \in$ For and $c(A)=0$. Then by Definition $6.1 A$ is ed, hence by Definition 6.3 is in cnf. By Fact 5.3, for every $C \in$ For we have, $\vdash_{\mathrm{S}} C \equiv C$.

Inductive hypothesis. Let $n \in \mathbb{N}$. Suppose for every $C \in$ For, if $c(C) \leq n$, then the fact holds for $C$.

Inductive step. Let $A \in$ For and $c(A)=n+1$. Then:

- Let $A:=\neg D$. By the inductive hypothesis for some $B \in$ For which is in cnf we have that: $\vdash_{\mathrm{S}} D \equiv B(1)$ and for every $C \in$ For, $\vdash_{\mathrm{S}}(D \leftrightarrow$ $C) \equiv(B \leftrightarrow C)(2)$.

$B$ is in cnf. Hence, by Remark 6.2 and $6.4: B:=\left(B_{1_{1}} \vee \ldots \vee\right.$ $\left.B_{n_{1}}\right) \wedge \ldots \wedge\left(B_{1_{m}} \vee \ldots \vee B_{n_{m}}\right)$, where for every $i \leq n$ and $j \leq m$, $B_{i_{j}} \in \mathrm{Li} \cup \mathrm{rPI} \cup \mathrm{nrPl}$. Let $\bar{B}:=\left(B_{1_{1}}^{\prime} \vee B_{1_{2}}^{\prime} \vee \ldots \vee B_{1_{m}}^{\prime}\right) \wedge\left(B_{2_{1}}^{\prime} \vee B_{2_{2}}^{\prime} \vee\right.$ $\left.\ldots \vee B_{2_{m}}^{\prime}\right) \wedge \ldots \wedge\left(B_{n_{1}}^{\prime} \vee B_{n_{2}}^{\prime} \vee \ldots \vee B_{n_{m}}^{\prime}\right)$. Hence, $\bar{B}$ is in cnf. 
Let us notice that, by Fact 5.3: $\vdash_{\mathrm{S}} \neg B \equiv\left[\left(B_{1_{1}}^{\prime} \wedge \ldots \wedge B_{n_{1}}^{\prime}\right) \vee \ldots \vee\right.$ $\left.\left(B_{1_{m}}^{\prime} \wedge \ldots \wedge B_{n_{m}}^{\prime}\right)\right](3)$ and $\vdash_{\mathrm{S}}\left[\left(B_{1_{1}}^{\prime} \wedge \ldots \wedge B_{n_{1}}^{\prime}\right) \vee \ldots \vee\left(B_{1_{m}}^{\prime} \wedge \ldots \wedge\right.\right.$ $\left.\left.B_{n_{m}}^{\prime}\right)\right] \equiv \bar{B}(4)$. Hence, by Fact 5.3 (transitivity of $\equiv$ ), (1), (3), (4) and (MP) we get: $\vdash_{\mathrm{S}} \neg D \equiv \bar{B}$.

Let us notice that, for every $C \in$ For: $\vdash_{\mathrm{S}}[(D \rightarrow C) \equiv(B \rightarrow C)] \supset$ $[(\neg D \rightarrow C) \equiv(\neg B \rightarrow C)](5)$ and $\vdash_{\mathrm{S}}(\neg B \rightarrow C) \equiv(\bar{B} \rightarrow C)(6)$. Hence, by Fact 5.3 (transitivity of $\equiv),(2),(5),(6)$ and (MP) we get: $\vdash_{\mathrm{S}}(\neg D \rightarrow C) \equiv(\bar{B} \leftrightarrow C)$.

- Let $A:=D \wedge E$. By the inductive hypothesis for some $B_{0}, B_{1} \in$ For which are in cnf we have that: $\vdash_{\mathrm{S}} D \equiv B_{0}(1)$, for every $C \in$ For, $\vdash_{\mathrm{S}}(D \rightarrow C) \equiv\left(B_{0} \rightarrow C\right)(2), \vdash_{\mathrm{S}} E \equiv B_{1}(3)$ and for every $C \in$ For, $\vdash_{\mathrm{S}}(E \rightarrow C) \equiv\left(B_{1} \rightarrow C\right)(4)$.

$B_{0}, B_{1}$ are in cnf. Hence, by Remark 6.2 and 6.4: $B_{0}:=\left(C_{1_{1}} \vee \ldots \vee\right.$ $\left.C_{n_{1}}\right) \wedge \ldots \wedge\left(C_{1_{m}} \vee \ldots \vee C_{n_{m}}\right)$, where for every $i \leq n$ and $j \leq m$, $C_{i_{j}} \in \mathrm{Li} \cup \mathrm{rPI} \cup \mathrm{nrPI}$ and $B_{1}:=\left(D_{1_{1}} \vee \ldots \vee D_{k_{1}}\right) \wedge \ldots \wedge\left(D_{1_{l}} \vee \ldots \vee D_{k_{l}}\right)$, where for every $i \leq k$ and $j \leq l, D_{i_{j}} \in \mathrm{Li} \cup \mathrm{rPI} \cup \mathrm{nrPl}$. Let $B_{2}:=$ $\left(C_{1_{1}} \vee \ldots \vee C_{n_{1}}\right) \wedge \ldots \wedge\left(C_{1_{m}} \vee \ldots \vee C_{n_{m}}\right) \wedge\left(D_{1_{1}} \vee \ldots \vee D_{k_{1}}\right) \wedge \ldots \wedge$ $\left(D_{1_{l}} \vee \ldots \vee D_{k_{l}}\right)$. Hence, $B_{2}$ is in cnf.

Let us notice that, by Fact $5.3: \vdash_{\mathrm{S}}\left(D \equiv B_{0}\right) \supset\left[\left(E \equiv B_{1}\right) \supset\right.$ $\left.\left((D \wedge E) \equiv\left(B_{0} \wedge B_{1}\right)\right)\right](5)$ and $\vdash_{\mathrm{S}}\left(B_{0} \wedge B_{1}\right) \equiv B_{2}$ (6). Hence, by Fact 5.3 (transitivity of $\equiv$ ), (1), (3), (5), (6) and (MP) we get: $\vdash_{\mathrm{S}}(D \wedge E) \equiv B_{2}$.

Let us notice that, for every $C \in$ For: $\vdash_{\mathrm{S}}\left[(D \rightarrow C) \equiv\left(B_{0} \rightarrow C\right)\right] \supset$ $\left[\left((E \rightarrow C) \equiv\left(B_{1} \rightarrow C\right)\right) \supset\left(((D \wedge E) \rightarrow C) \equiv\left(\left(B_{0} \wedge B_{1}\right) \rightarrow C\right)\right)\right](7)$ and $\vdash_{\mathrm{S}}\left(\left(B_{0} \wedge B_{1}\right) \rightarrow C\right) \equiv\left(B_{2} \rightarrow C\right)$ (8). Therefore, by Fact 5.3 (transitivity of $\equiv),(2),(4),(7),(8)$ and (MP) we get: $\vdash_{\mathrm{S}}((D \wedge E) \rightarrow$ $C) \equiv\left(B_{2} \rightarrow C\right)$.

- Let $A:=D \rightarrow E$. By the inductive hypothesis for some $B_{0}, B_{1} \in$ For which are in cnf we have that: $\vdash_{\mathrm{S}} D \equiv B_{0}(1)$, for every $C \in$ For, $\vdash_{\mathrm{S}}(D \rightarrow C) \equiv\left(B_{0} \rightarrow C\right)(2), \vdash_{\mathrm{S}} E \equiv B_{1}(3)$ and for every $C \in$ For, $\vdash_{\mathrm{S}}(E \rightarrow C) \equiv\left(B_{1} \rightarrow C\right)(4)$.

$B_{0}, B_{1}$ are in cnf. Hence, by Remark 6.2 and $6.4: B_{0}:=\left(C_{1_{1}} \vee\right.$ $\left.\ldots \vee C_{n_{1}}\right) \wedge \ldots \wedge\left(C_{1_{m}} \vee \ldots \vee C_{n_{m}}\right)$, where for every $i \leq n$ and $j \leq m, C_{i_{j}} \in \mathrm{Li} \cup \mathrm{rPI} \cup \mathrm{nrPl}$. Also by Remark 6.2 and 6.4: $B_{1}:=$ $\left(D_{1_{1}} \vee \ldots \vee D_{k_{1}}\right) \wedge \ldots \wedge\left(D_{1_{l}} \vee \ldots \vee D_{k_{l}}\right)$, where for every $i \leq k$ and $j \leq l$, $D_{i_{j}} \in$ LiUrPIUnrPI. Let $\overline{B_{0}}:=\left(C_{1_{1}}^{\prime} \vee \ldots \vee C_{1_{m}}^{\prime}\right) \wedge \ldots \wedge\left(C_{n_{1}}^{\prime} \vee \ldots \vee C_{n_{m}}^{\prime}\right)$. Hence, $\overline{B_{0}}$ is in cnf. Let $B_{2}:=\left(C_{1_{1}}^{\prime} \vee \ldots \vee C_{1_{m}}^{\prime} \vee D_{1_{1}} \vee \ldots \vee D_{k_{1}}\right) \wedge$ 
A Post-Style Proof of Completeness Theorem for Symmetric Relatedness. . 211

$\left(C_{1_{1}}^{\prime} \vee \ldots \vee C_{1_{m}}^{\prime} \vee D_{1_{2}} \vee \ldots \vee D_{k_{2}}\right) \wedge \ldots \wedge\left(C_{n_{1}}^{\prime} \vee \ldots \vee C_{n_{m}}^{\prime} \vee D_{1_{l}} \vee \ldots \vee D_{k_{l}}\right)$. Formula $B_{2}$ is also in cnf.

Let us notice that, by the Fact 5.3: $\vdash_{\mathrm{S}}\left(\neg B_{0} \vee B_{1}\right) \equiv\left(\overline{B_{0}} \vee B_{1}\right)$ (5) and $\vdash_{\mathrm{S}}\left(\overline{B_{0}} \vee B_{1}\right) \equiv B_{2}(6)$. Hence, by Fact 5.3 (transitivity of $\equiv$ ), (5), (6) and (MP) we get: $\vdash_{\mathrm{S}}\left(\neg B_{0} \vee B_{1}\right) \equiv B_{2}(7)$.

For every $i \leq n$ and $j \leq m, C_{i_{j}} \in \mathrm{Li} \cup \mathrm{rPI} \cup \mathrm{nrPI}$ and for every $i \leq k$ and $j \leq l, B_{i_{j}} \in \mathrm{Li} \cup \mathrm{rPI} \cup \mathrm{nrPI}$. Let $B_{3}=\left(C_{1_{1}}^{a} \rightarrow D_{1_{1}}^{a}\right) \vee\left(C_{1_{1}}^{a} \rightarrow\right.$ $\left.D_{1_{1}}^{c}\right) \vee\left(C_{1_{1}}^{c} \leftrightarrow D_{1_{1}}^{a}\right) \vee\left(C_{1_{1}}^{c} \rightarrow D_{1_{1}}^{c}\right) \vee \ldots \vee\left(C_{n_{m}}^{a} \rightarrow D_{k_{l}}^{a}\right) \vee\left(C_{n_{m}}^{a} \rightarrow\right.$ $\left.D_{k_{l}}^{c}\right) \vee\left(C_{n_{m}}^{c} \rightarrow D_{k_{l}}^{a}\right) \vee\left(C_{n_{m}}^{c} \rightarrow D_{k_{l}}^{c}\right)$. Hence, $B_{3}$ is ed.

Let us notice that: $\vdash_{\mathrm{S}}\left(B_{0} \uparrow \rightarrow B_{1}\right) \equiv B_{3}$ (8). Hence, by Fact 5.3, (7), (8) and (MP) we get: $\vdash_{\mathrm{S}}\left[\left(\neg B_{0} \vee B_{1}\right) \wedge\left(B_{0} \rightarrow B_{1}\right)\right] \equiv\left(B_{2} \wedge B_{3}\right)(9)$. Let $B_{4}:=\left(C_{1_{1}}^{\prime} \vee \ldots \vee C_{1_{m}}^{\prime} \vee D_{1_{1}} \vee \ldots \vee D_{k_{1}}\right) \wedge\left(C_{1_{1}}^{\prime} \vee \ldots \vee C_{1_{m}}^{\prime} \vee\right.$ $\left.D_{1_{2}} \vee \ldots \vee D_{k_{2}}\right) \wedge \ldots \wedge\left(C_{n_{1}}^{\prime} \vee \ldots \vee C_{n_{m}}^{\prime} \vee D_{1_{l}} \vee \ldots \vee D_{k_{l}}\right) \wedge\left[\left(C_{1_{1}}^{a} \rightarrow\right.\right.$ $\left.D_{1_{1}}^{a}\right) \vee\left(C_{1_{1}}^{a} \rightarrow D_{1_{1}}^{c}\right) \vee\left(C_{1_{1}}^{c} \rightarrow D_{1_{1}}^{a}\right) \vee\left(C_{1_{1}}^{c} \rightarrow D_{1_{1}}^{c}\right) \vee \ldots \vee\left(C_{n_{m}}^{a} \leftrightarrow\right.$ $\left.\left.D_{k_{l}}^{a}\right) \vee\left(C_{n_{m}}^{a} \rightarrow D_{k_{l}}^{c}\right) \vee\left(C_{n_{m}}^{c} \rightarrow D_{k_{l}}^{a}\right) \vee\left(C_{n_{m}}^{c} \rightarrow D_{k_{l}}^{c}\right)\right]$. Therefore, $B_{4}$ is in cnf.

Let us notice that, by Fact 5.3: $\vdash_{\mathrm{S}}\left(B_{2} \wedge B_{3}\right) \equiv B_{4}$ (10). Hence, by Fact 5.3 (transitivity of $\equiv),(9),(10)$ and (MP) we get: $\vdash_{\mathrm{S}}\left[\left(\neg B_{0} \vee\right.\right.$ $\left.\left.B_{1}\right) \wedge\left(B_{0} \uparrow B_{1}\right)\right] \equiv B_{4}(11)$. We also have that, for every $C \in$ For, $\vdash_{\mathrm{S}}\left(\left(B_{0} \wedge B_{1}\right) \rightarrow C\right) \equiv\left(B_{4} \rightarrow C\right)(12)$.

Let us notice that: $\vdash_{\mathrm{S}}\left[\left(E \rightarrow B_{0}\right) \equiv\left(B_{1} \rightarrow B_{0}\right)\right] \supset\left[\left(B_{0} \rightarrow E\right) \equiv\right.$ $\left.\left(B_{0} \leftrightarrow B_{1}\right)\right]$ (13). Hence, by Fact 5.3 (transitivity of $\left.\equiv\right)$, (2), (4), (13), and (MP) we get: $\vdash_{\mathrm{S}}(D \rightarrow E) \equiv\left(B_{0} \rightarrow B_{1}\right)(14)$.

Let us notice that: $\vdash_{\mathrm{S}}\left[(D \rightarrow E) \equiv\left(B_{0} \rightarrow B_{1}\right)\right] \supset\left[\left(D \equiv B_{0}\right) \supset\right.$ $\left.\left(\left(E \equiv B_{1}\right) \supset\left((D \rightarrow E) \equiv\left(\left(\neg B_{0} \vee B_{1}\right) \wedge\left(B_{0} \rightarrow B_{1}\right)\right)\right)\right)\right]$ (15). Hence, by (1), (3), (14), (15) and (MP): $\vdash_{\mathrm{S}}(D \rightarrow E) \equiv\left(\left(\neg B_{0} \vee B_{1}\right) \wedge\left(B_{0} \rightarrow\right.\right.$ $\left.B_{1}\right)$ ) (16). Hence, by Fact 5.3 (transitivity of $\left.\equiv\right),(11),(16)$ and (MP) we get: $\vdash_{\mathrm{S}}(D \rightarrow E) \equiv B_{4}$.

Let us notice that, by Fact 5.3: $\vdash_{\mathrm{S}}\left[(D \rightarrow C) \equiv\left(B_{0} \rightarrow C\right)\right] \supset[((E \leftrightarrow$ $\left.C) \equiv\left(B_{1} \rightarrow C\right)\right) \supset((D \rightarrow C) \vee(E q C)) \equiv\left(\left(B_{0} \rightarrow C\right) \vee\left(B_{1} \rightarrow\right.\right.$ $C)))](16)$. For every $C \in$ For we have: $\vdash_{\mathrm{S}}((D \rightarrow E) \rightarrow C) \equiv((D \rightarrow$ $C) \vee(E \leftrightarrow C))(17)$ and $\vdash_{\mathrm{S}}\left(\left(B_{0} \rightarrow C\right) \vee\left(B_{1} \rightarrow C\right)\right) \equiv\left(\left(B_{0} \wedge B_{1}\right) \rightarrow\right.$ $C)$ (18). Hence, by Fact 5.3 (transitivity of $\equiv)$, (2), (4), (16), (17), (18) and (MP) we get: $\vdash_{\mathrm{S}}((D \rightarrow E) \rightarrow C) \equiv\left(\left(B_{0} \wedge B_{1}\right) \rightarrow C\right)$ (19). Hence, by Fact 5.3 (transitivity of $\equiv),(12),(19)$ and (MP) we get: $\vdash_{\mathrm{S}}((D \rightarrow E) \rightarrow C) \equiv\left(B_{4} \rightarrow C\right)$.

Lemma 6.8. Let $A:=B_{1} \vee \ldots \vee B_{n}$ be ed $(n \in \mathbb{N})$. Then: $\models_{S} A$ iff at least 
one of the following conditions hold:

(1) $B_{k}:=p_{i}$ and $B_{l}:=\neg p_{i}$, for some $k, l \leq n$ and $i \in \mathbb{N}$

(2) $B_{k}:=p_{i} \rightarrow p_{i}$, for some $k \leq n$ and $i \in \mathbb{N}$

(3) $B_{k}:=p_{i} \rightarrow p_{j}$ and $B_{l}:=\neg\left(p_{i} \rightarrow p_{j}\right)$, for some $k, l \leq n$ and $i, j \in \mathbb{N}$.

Proof: Assume all hypothesis.

„” Suppose that non of the conditions (1)-(3) holds $(\star)$. We define a model $\mathfrak{M}=\langle v, \mathbf{R}\rangle$ in the following way:

1. Let $i \in \mathbb{N}$ we put:

$$
v\left(p_{i}\right)= \begin{cases}1, & \text { if } \neg p_{i} \in \operatorname{sub}(A), \\ 0, & \text { if } p_{i} \in \operatorname{sub}(A) .\end{cases}
$$

2. Let $i, j \in \mathbb{N}$ and $i \neq j$. Let $\mathrm{Q} \subseteq \mathrm{PI} \times \mathrm{PI}$ be the smallest relation which fulfils the following conditions:

- $p_{i} \leftrightarrow p_{j} \in \operatorname{sub}(A) \Longrightarrow \sim \mathrm{Q}\left(p_{i}, p_{j}\right)$

- $\neg\left(p_{i} \rightarrow p_{j}\right) \in \operatorname{sub}(A) \Longrightarrow \mathrm{Q}\left(p_{i}, p_{j}\right)$

- $\mathrm{Q}\left(p_{i}, p_{i}\right)$

- $\mathrm{Q}\left(p_{i}, p_{j}\right)$ iff $\mathrm{Q}\left(p_{j}, p_{i}\right)$.

$\mathrm{Q}$ is obviously reflexive and symmetric. We extend $\mathrm{Q}$ on For in the following way for every $A, B \in$ For:

$$
\mathrm{R}(A, B) \text { iff } \exists_{x \in \mathrm{pl}(A)} \exists_{y \in \mathrm{pl}(B)} \mathrm{Q}(x, y) .
$$

By Fact $3.2 \mathrm{R}$ is srr. Let $i, j \in \mathbb{N}$ and $i \neq j$. Let us consider the following cases:

- Suppose $B_{k}:=p_{i}$, for some $k \leq n$. By the definition of $v_{\mathfrak{M}}$ we get $\mathfrak{M} \not \nvdash_{\mathrm{S}} p_{n}$.

- Suppose $B_{k}:=\neg p_{n}$, for some $k \leq n$. By the definition of $v_{\mathfrak{M}}$ we get $\mathfrak{M} \forall_{\mathrm{S}} \neg p_{n}$.

- By ( $\star$ ) it is excluded that: $B_{k}=p_{i}$ and $B_{l}:=\neg p_{i}$, for some $k, l \leq n$.

- Suppose $B_{k}:=p_{i} \rightarrow p_{j}$, for some $k \leq n$. By the definition of $\mathrm{R}_{\mathfrak{M}}$ we get $\mathfrak{M} \mid \nvdash_{\mathrm{S}} p_{i} \rightarrow p_{j}$.

- Suppose $B_{k}:=\neg\left(p_{i} \rightarrow p_{j}\right)$, for some $k \leq n$. By the definition of $\mathrm{R}_{\mathfrak{M}}$ we get $\mathfrak{M} \not \models_{\mathrm{S}} \neg\left(p_{i} \uparrow \rightarrow p_{j}\right)$.

- By $(\star)$ it is excluded that: $B_{k}:=p_{i} \leftrightarrow p_{j}$, for some $k \leq n$ and it is excluded that: $B_{k}:=p_{i} \rightarrow p_{j}$ and $B_{l}:=\neg\left(p_{i} \uparrow p_{j}\right)$, for some $k, l \leq n$.

Therefore, $\mathfrak{M} \not \nvdash_{\mathrm{S}} A$, so by Definition $4.4 \not \nvdash_{\mathrm{S}} A$. 
„£" Suppose that at least one of the conditions (1)-(3) holds. Let $\mathfrak{M} \in \mathcal{M}$. If condition (1) or (3) holds then, by Definition $4.2, \mathfrak{M} \models A$. If condition (2) holds and $B_{k}:=p_{i} \leftrightarrow p_{i}$, for some $k \leq n$ and $i \in \mathbb{N}$. By (re) $\mathrm{R}_{\mathfrak{M}}\left(p_{i}, p_{i}\right)$. Therefore, by Fact $4.3, \mathfrak{M} \models p_{i} \rightarrow p_{i}$. Hence, by Definition $4.2, \mathfrak{M} \mid=A$. Therefore, $\mathfrak{M} \models_{\mathrm{S}} A$. Thus, by Definition $4.4, \models_{\mathrm{S}} A$.

Lemma 6.9. Let $A:=B_{1} \wedge \ldots \wedge B_{n}$ be in $\operatorname{cnf}(n \in \mathbb{N})$. Then: $\models_{S} A$ iff $\models_{S}$ $B_{k}$, for every $k \leq n$.

Proof: Assume all hypothesis. By Definition 4.2: $\models_{\mathrm{S}} B_{1} \wedge \ldots \wedge B_{n}$ iff $\models_{\mathrm{S}}$ $B_{k}$, for every $k \leq n$.

\section{Completeness theorem for logic $\mathrm{S}$}

Theorem 7.1 (Completeness theorem for logic S). Let $A \in$ For. Then: $\models_{S} A \Longrightarrow{ }_{S} A$.

Proof: Let $A \in$ For. Suppose $\models_{\mathrm{S}} A(1)$. By Fact 6.7 for some $B \in$ For in cnf we have $\vdash_{\mathrm{S}} A \equiv B(2)$. By Fact 5.2 we get $\left.\right|_{\mathrm{S}} A \equiv B(3)$. Hence, by (1) and (3), $\models \models_{\mathrm{S}} B$. Moreover, $B$ is in cnf. Let $n \in \mathbb{N}$ and $B:=B_{1} \wedge \ldots \wedge B_{n}$, where for every $i \leq n, B_{i}$ is ed. Let $i \leq n$, by Lemma $6.9, \models_{\mathrm{S}} B_{i}$. We also have that $B_{i}:=C_{1_{i}} \vee \ldots \vee C_{m_{i}}$, for some $m \in \mathbb{N}$, and for every $k \leq m$, $C_{k_{i}} \in \mathrm{Li} \cup \mathrm{rPI} \cup \mathrm{nrPI}$. By Lemma 6.8 at least one of the following conditions holds:

(a) $C_{k_{i}}:=p_{j}$ and $C_{l_{i}}:=\neg p_{j}$, for some $k, l \leq m$ and $j \in \mathbb{N}$

(b) $C_{k_{i}}:=p_{j} \rightarrow p_{j}$, for some $k \leq m$ and $j \in \mathbb{N}$

(c) $C_{k_{i}}:=p_{j} \leftrightarrow p_{h}$ and $C_{l_{i}}:=\neg\left(p_{j} \leftrightarrow p_{h}\right)$, for some $k, l \leq m$ and $i, h \in \mathbb{N}$.

Suppose condition (a) holds and $B_{i}:=p_{j} \vee \neg p_{j} \vee C$, where $C$ is not important part of $B_{i}$. Let us notice that $\vdash_{\mathrm{S}} p_{j} \vee \neg p_{j}$ (4). Moreover, for every $D, E \in$ For we have that $\vdash_{\mathrm{S}} D \rightarrow(D \vee E)$ (5). Hence, by (4) and $(5), \vdash \mathrm{S} B_{i}$.

Suppose condition (b) holds and $B_{i}:=p_{j} \rightarrow p_{j} \vee C$, where $C$ is not important part of $B_{i}$. Let us notice that $\vdash_{\mathrm{S}} p_{j} \uparrow p_{j}$. We reason as in the case of condition (a).

Suppose condition (c) holds and $B_{i}:=\left(p_{j} \rightarrow p_{h}\right) \vee \neg\left(p_{j} \rightarrow p_{h}\right) \vee C$, where $C$ is not important part of $B_{i}$. Let us notice that $\vdash_{\mathrm{S}}\left(p_{j} \rightarrow p_{h}\right) \vee$ $\neg\left(p_{j} q p_{h}\right)$. We reason as in the case of condition (a). 
Hence, $\vdash_{\mathrm{S}} B_{i}$, for every $i \leq n(6)$. Let us also note that $\vdash_{\mathrm{S}} D \rightarrow(E \rightarrow$ $(D \wedge E))(7)$. By $(6)$ and $(7)$ we get $\vdash_{\mathrm{S}} B_{1} \wedge \ldots \wedge B_{n}$. Hence, $\vdash_{\mathrm{S}} B$. And therefore, by (2), $\vdash_{\mathrm{S}} A$.

\section{References}

[1] R. L. Epstein, Relatedness and Implication, Philosophical Studies, Vol. 36:2 (1979), pp. 137-173.

[2] R. L. Epstein, (with the assistance and collaboration of: W. A. Camielli, I. M. L. D'Ottaviano, S. Krajewski, R. D. Maddux), The Semantic Foundtations of Logic. Volume 1: Propositional Logics, Springer Science+Business Media, Dordrecht (1990).

[3] T. Jarmużek and B. Kaczkowski, On some Logic with a Relation Imposed on Formulae: Tableau System $\mathcal{F}$, Bulletin of the Section of Logic, Vol. 43:1/2 (2014), pp. 53-72.

[4] S. Krajewski, One or Many Logics? (Epstein's Set-Assignement Semantics for Logical Calculi), The Journal of Non-Classical Logic 8:1 (1991), pp. 7-33.

[5] S. Krajewski, On Relatedness Logic of Richard L. Epstein, Bulletin of the Section of Logic, Vol. 11:1/2 (1982), pp. 24-30.

[6] J. B. Rosser, Logic for Mathematicians, McGraw-Hill, New York (1953).

[7] D. Walton, Philosophical Basis of Relatedness Logic, Philosophical Studies, Vol. 36:2 (1979), pp. 115-136.

Department of Logic

Nicolaus Copernicus University

ul. Fosa Staromiejska 1a, 87-100, Toruń

Poland

e-mail: matklon@doktorant.umk.pl 